Article

\title{
Murine Anorectic Response to Deoxynivalenol (Vomitoxin) Is Sex-Dependent
}

\author{
Erica S. Clark ${ }^{1,2}$, Brenna M. Flannery ${ }^{1,2}$ and James J. Pestka ${ }^{1,2,3, *}$
}

1 Department of Food Science and Human Nutrition, Michigan State University, East Lansing, MI 48824, USA; E-Mails: clarke27@msu.edu (E.S.C.); flanne12@msu.edu (B.M.F.)

2 Center for Integrative Toxicology, Michigan State University, East Lansing, MI 48824, USA

3 Department of Microbiology and Molecular Genetics, Michigan State University, East Lansing, MI 48824, USA

* Author to whom correspondence should be addressed; E-Mail: pestka@msu.edu;

Tel.: +1-517-353-1709; Fax: +1-517-353-8963.

Academic Editor: Paul Turner

Received: 5 June 2015 / Accepted: 17 July 2015 / Published: 29 July 2015

\begin{abstract}
Deoxynivalenol (DON, vomitoxin), a common trichothecene mycotoxin found in cereal foods, dysregulates immune function and maintenance of energy balance. The purpose of this study was to determine if sex differences are similarly evident in DON's anorectic responses in mice. A bioassay for feed refusal, previously developed by our lab, was used to compare acute i.p. exposures of 1 and $5 \mathrm{mg} / \mathrm{kg}$ bw DON in C57BL6 mice. Greater anorectic responses were seen in male than female mice. Male mice had higher organ and plasma concentrations of DON upon acute exposure than their female counterparts. A significant increase in IL-6 plasma levels was also observed in males while cholecystokinin response was higher in females. When effects of sex on food intake and body weight changes were compared after subchronic dietary exposure to $1,2.5$, and $10 \mathrm{ppm} \mathrm{DON}$, males were found again to be more sensitive. Demonstration of male predilection to DON-induced changes in food intake and weight gain might an important consideration in future risk assessment of DON and other trichothecenes.
\end{abstract}

Keywords: trichothecene; mycotoxin; anorexia; weight loss; IL-6; mouse; sex dependence 


\section{Introduction}

Deoxynivalenol (DON, vomitoxin) is a trichothecene mycotoxin produced by the fungus Fusarium graminearum that contaminates corn, wheat, and barley [1]. DON is highly resistant to heat processing and can enter human and animal food. A recent study reported high levels of DON contamination in corn $(76 \%)$ and wheat $(79 \%)$ samples obtained from North America, suggesting that exposure to this mycotoxin is frequent [2]. In experimental animals, adverse effects of acute exposure include symptoms of gastrointestinal illness while chronic exposure can lead to growth retardation and immunotoxic effects [3-6]. In species capable of emesis (e.g. pig, mink), DON rapidly induces vomiting $[7,8]$. Rodents, however, are incapable of vomiting and instead exhibit feed refusal following exposure to this toxin $[9,10]$.

Previous investigations suggest that male animals are more sensitive than female animals to the adverse effects from DON consumption [11-14]. Studies in swine have reported greater sensitivities in males to weight suppression and food intake than females $[12,15]$. An investigation conducted in ICR mice found that males fed DON containing diets showed greater growth depression and lower food intake than female mice [13]. The same study also reported that the amount of DON consumed by body weight was lower in male mice (1.49 $\mathrm{mg} \mathrm{DON} / \mathrm{kg}$ bw/day) than in female mice (1.59 $\mathrm{mg} \mathrm{DON} / \mathrm{kg}$ bw/day) further supporting the contention that the response to this toxin differs by sex. A two-year feeding study in $\mathrm{B} 6 \mathrm{C} 3 \mathrm{~F} 1$ mice found that males fed DON diets consumed less food than females, though this did not lead to a significant reduction in body weight [4]. Finally, previous investigations by our laboratory identifying a male predilection to DON induced IgA nephropathy also reported greater weight suppression in male mice than female mice $[11,14,16]$.

DON-induced feed refusal have been linked to the induction of proinflammatory cytokines (i.e., IL-1 $\beta$, IL-6, and TNF- $\alpha$ ), which are known to have anorectic actions in animals and humans [17-19]. DON exposure has previously been shown to induce these proinflammatory cytokine expressions in the female mouse model [20,21]. Since immune dysregulation of IgA is greater in male mice than female mice, comparison of sex differences in these cytokines is also of interest.

Elevation of the satiety hormones cholecystokinin (CCK) and peptide YY (PYY) have also been observed upon DON exposure [22,23]. CCK is a hormone that is secreted by I cells within the small intestine and increases the expression of anorexigenic peptides including cocaine and amphetamine regulated transcript (CART) [24]. PYY is a 36-amino acid peptide that also decreases food intake by increasing the expression of anorexigenic peptides and is secreted by the L cells of the colon and ileum [25]. While previous studies have explored elevation of these hormones in female animals following DON exposure, the effect of DON on satiety hormones in males has yet to be addressed.

The aims of this study were to evaluate and characterize murine sex differences in food refusal response after acute and dietary DON exposure. Study 1 addressed sex differences in feed refusal response to acute i.p. DON exposure. Study 2 compared DON tissue concentrations, as well as proinflammatory cytokine and satiety hormone responses to acute DON exposure in males and females. The objective of Study 3 was to compare sex differences in food intake and body weight suppression upon exposure to dietary DON. The results indicate that male mice were more sensitive than female mice to acute i.p. and dietary DON exposure and that these differences correspond to slower toxin organ clearance and an increased IL-6 response with acute i.p. exposure. 


\section{Results and Discussion}

\subsection{Study 1}

Feed Refusal upon Acute DON Exposure Is Greater in Males than Females

The effects of acute i.p. exposure to DON on food intake were compared in male and female mice over a period of $36 \mathrm{~h}$ (Figure 1). Male mice treated with $1 \mathrm{mg} / \mathrm{kg}$ bw DON ate $45 \%$ less food then control males at $6 \mathrm{~h}$ post-injection (PI) (Figure 2). After $6 \mathrm{~h}$, male mice showed recovery with cumulative food intake nearly reaching that of controls $36 \mathrm{~h} \mathrm{PI}$. In contrast, female mice treated with $1 \mathrm{mg} / \mathrm{kg}$ bw DON ate $24 \%$ less food than female control mice at $6 \mathrm{~h}$ PI and began to show recovery in food intake at $5 \mathrm{~h}$ PI. Male mice treated with $5 \mathrm{mg} / \mathrm{kg}$ bw exhibited the greatest difference in food intake from the control males between 7 and $12 \mathrm{~h} \mathrm{PI}$, consuming 7\% of food intake compared to control males. Food intake in males exposed to $5 \mathrm{mg} / \mathrm{kg}$ bw DON started to increase after $12 \mathrm{~h} \mathrm{PI}$, however, at $36 \mathrm{~h}$ PI males had consumed only $82 \%$ of what control males had eaten. Conversely, female mice treated with $5 \mathrm{mg} / \mathrm{kg}$ bw DON started to show recovery in food intake at $7 \mathrm{~h}$ after DON treatment and had eaten $33 \%$ of that by control females. At the final time point of $36 \mathrm{~h} \mathrm{PI}$, food intake of female mice treated with $5 \mathrm{mg} / \mathrm{kg}$ bw DON had almost completely recovered with the animals having cumulatively consumed $95 \%$ of the food intake of control females.

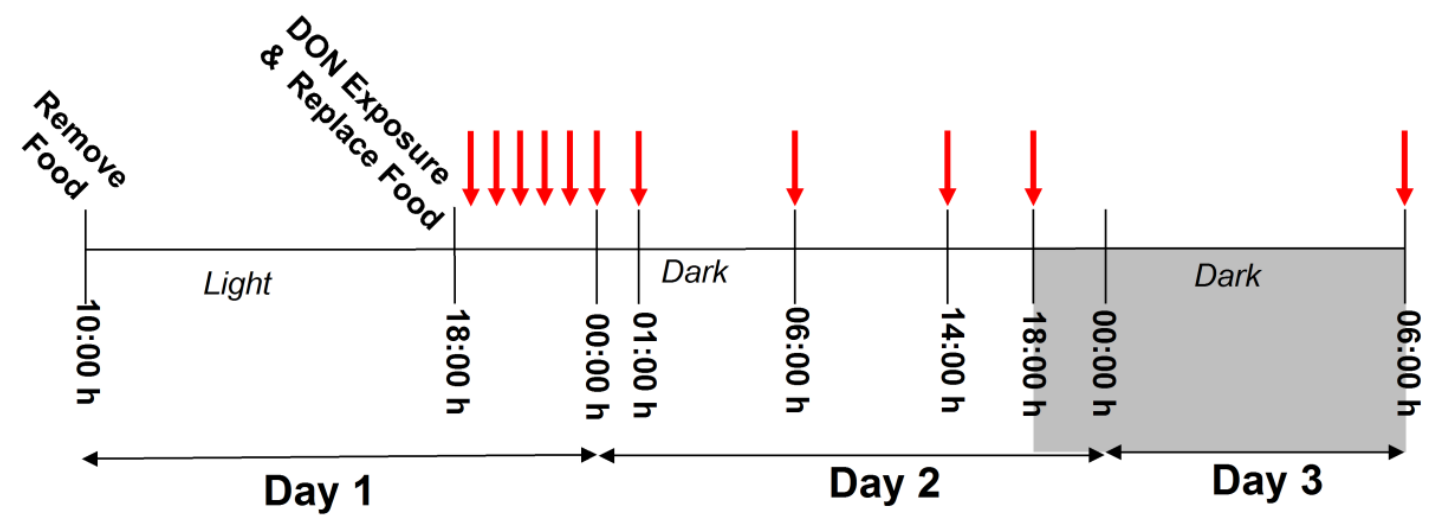

Figure 1. Study 1 experimental design. On Day 1, mice were fasted from 10:00 a.m. to 6:00 p.m. then exposed to DON treatments or vehicle control. Food was immediately replaced following exposure and food measurements were recorded hourly from 1 to $7 \mathrm{~h}$ PI, and at 12, 20, 24, and $36 \mathrm{~h}$ PI as indicated by arrows.

While this is the first comparison of murine sex differences in DON-induced anorectic effects, acute feed refusal has been previously described in $\mathrm{B} 6 \mathrm{C} 3 \mathrm{~F} 1$ female mice. Flannery et al. $[10,22]$ observed that by $4 \mathrm{~h}$ food intake had recovered in female B6C3F1 mice treated with $1 \mathrm{mg} / \mathrm{kg}$ bw DON i.p. In this study, we found that C57BL6 females at the same time point and dose continued to show feed refusal, consuming $37 \%$ food than control females. These findings are consistent with reports of greater sensitivity of C57BL6 females to body weight reduction and mortality with DON exposure in comparison to B6C3F1 females [11]. 


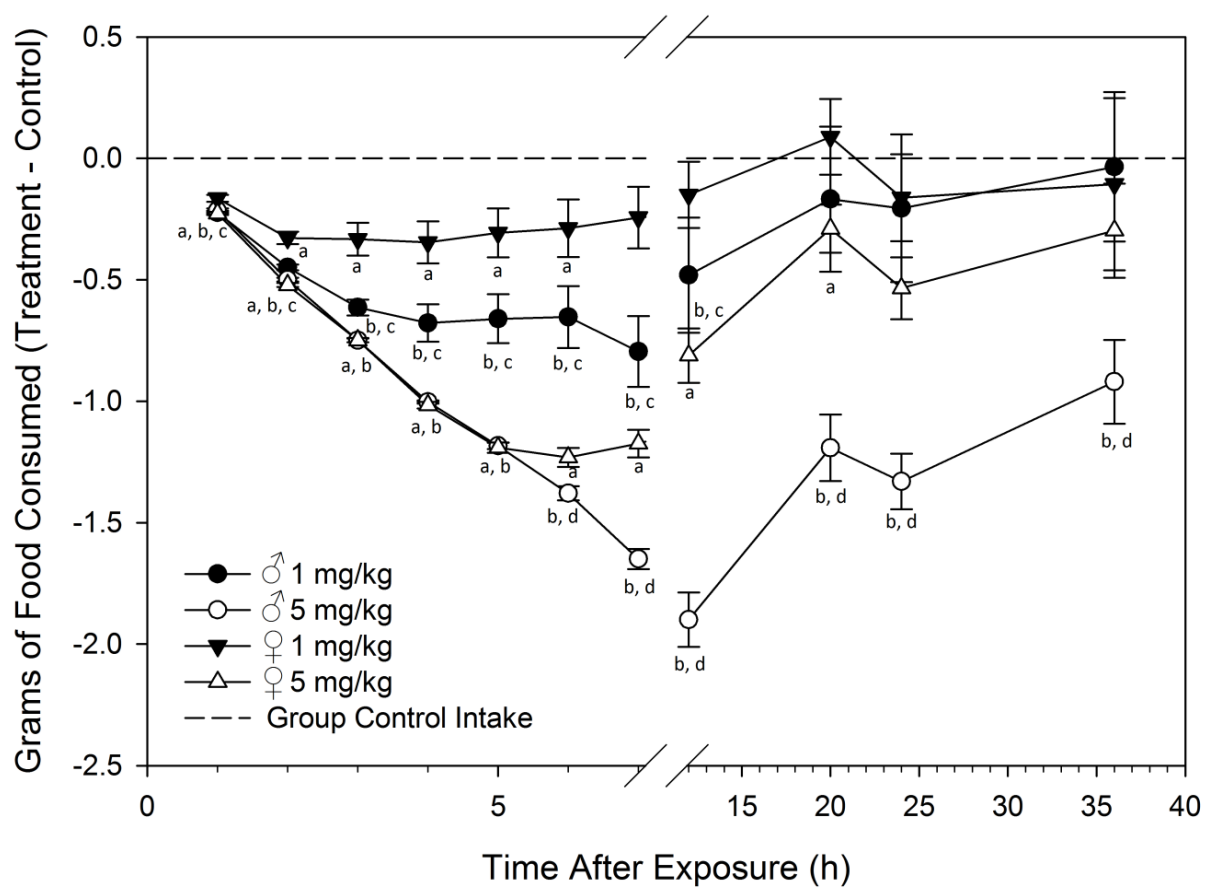

Figure 2. Male mice are more sensitive to DON-induced feed refusal than females following acute i.p. exposure. Food intake is cumulative at each time point and was normalized to group control average. Data are mean $\pm \operatorname{SEM}(n=10 / \mathrm{gp})$. Statistically significance differences are indicated as follows: $\mathrm{a}=\mathrm{DON}$-dosed female different from female control; $\mathrm{b}=\mathrm{DON}$-dosed male different from male control; $\mathrm{c}=$ male different from female at $1 \mathrm{mg} / \mathrm{kg}$ bw DON; and $\mathrm{d}=$ male different from female at $5 \mathrm{mg} / \mathrm{kg}$ bw DON $(p<0.05)$.

\subsection{Study 2}

\subsubsection{DON Organ Concentrations Are Higher in Male Mice after Acute DON Exposure}

Tissues were analyzed for DON following acute exposure to $1 \mathrm{mg} / \mathrm{kg}$ bw at 1,2 , and $4 \mathrm{~h} \mathrm{PI}$. DON concentrations were represented as DON equivalents, as the ELISA used to quantify DON is $100 \%$ cross-reactive with the DON metabolite deoxynivalenol-3-glucuronide (DONGlcA) (Supplementary Data, Figure S1). At 1 h PI, kidney, liver, and heart DON equivalent concentrations were higher in male mice than female mice (Table 1). Males had the highest concentration in kidney followed by the plasma. Female mice at this time point had the highest concentration of DON equivalents in the plasma, followed by the kidney. The rank order of DON equivalent concentrations in all other organs for males and females was: liver $>$ heart $>$ spleen $>$ brain. Similar tissue distribution patterns have previously been reported in B6C3F1 mice and pigs [26,27]. High concentrations of DON equivalents in the kidney of male mice at $1 \mathrm{~h}$ after DON treatment could indicate that male mice are excreting the toxin more slowly than female mice.

No differences in DON equivalent organ concentrations between males and females were observed at $2 \mathrm{~h}$ PI (data not shown). However, DON equivalent concentrations were significantly higher in males than females in all organs and plasma at $4 \mathrm{~h}$ post exposure, with the exception of the brain (Table 1). Interestingly, the brain showed the lowest change in DON equivalents from 1 to $4 \mathrm{~h}$, with the concentration remaining to be approximately $50 \%$ of the $1 \mathrm{~h}$ levels measured in both male and female 
mice while all organs had decreased DON levels from $7 \%$ to $14 \%$ of the $1 \mathrm{~h}$ measurement. Higher toxin levels remaining in the tissues of male mice could be a contributing factor to the increased feed refusal seen in males.

Table 1. DON equivalent concentrations are higher in male mice at 1 and $4 \mathrm{~h}$ post acute i.p. exposure to $1 \mathrm{mg} / \mathrm{kg}$ bw DON. DON equivalent concentrations are $\mathrm{nmol} / \mathrm{g}$. DON is reported as DON equivalents as the ELISA was found to be completely cross reactive with DON3GlcA. Control animals did not have detectable levels of DON (data not shown). Data are mean $\pm \operatorname{SEM}(n=5-6 / g p)$.

\begin{tabular}{c|cc|cc}
\hline \multirow{2}{*}{ Organ } & \multicolumn{4}{|c}{ DON Equivalents (nmol/g) } \\
\cline { 2 - 6 } & \multicolumn{2}{|c|}{$\mathbf{1 ~ h}$} & \multicolumn{2}{c}{$\mathbf{4 ~ h}$} \\
\hline & $\delta$ & + & $\hat{0}$ & $q$ \\
Kidney & $3.85 \pm 0.3 *$ & $2.21 \pm 0.2$ & $0.30 \pm 0.03 *$ & $0.17 \pm 0.01$ \\
Liver & $2.60 \pm 0.2^{p=0.09}$ & $1.86 \pm 0.3$ & $0.36 \pm 0.1 *$ & $0.14 \pm 0.02$ \\
Plasma & $2.72 \pm 0.2$ & $2.33 \pm 0.2$ & $0.20 \pm 0.01 *$ & $0.12 \pm 0.01$ \\
Heart & $2.24 \pm 0.1 *$ & $1.60 \pm 0.2$ & $0.19 \pm 0.01 *$ & $0.11 \pm 0.01$ \\
Spleen & $1.44 \pm 0.1$ & $1.16 \pm 0.2$ & $0.17 \pm 0.01 *$ & $0.10 \pm 0.01$ \\
Brain & $0.61 \pm 0.01$ & $0.64 \pm 0.04$ & $0.33 \pm 0.02$ & $0.30 \pm 0.05$ \\
\hline
\end{tabular}

$*$ indicates significantly different from female at specified time point $(p<0.05)$.

Overall, these data suggest that males may differ from females in absorption and/or clearance of DON. One possible metabolic difference is that males have a reduced capacity to glucuronidate, thus excrete the toxin. Sex differences have previously been identified in mRNA levels of UDP-glucuronosyltransferases (UGTs), the family of enzymes responsible for DON glucuronidation [28,29]. Analyzing possible sex differences in DON metabolism will be an important objective for future studies.

\subsubsection{Plasma IL-6 Is Higher in Male Mice than Female Mice upon Acute DON Exposure}

When the effects of acute exposure to $1 \mathrm{mg} / \mathrm{kg}$ bw DON on plasma levels of proinflammatory cytokines were determined, DON treated males and females both had significantly higher IL-6 plasma levels than their respective group control at 1 and $2 \mathrm{~h}$ PI (Figure 3). Plasma IL-6 concentrations were elevated 2.25 fold higher at $2 \mathrm{~h}$ after DON treatment in male mice when compared to female mice. The high increase in this anorectic cytokine could be another factor to male sensitivity to acute DON-induced anorexia.

Plasma TNF- $\alpha$ and IL-1 $\beta$ were not induced by DON (data not shown). These findings are consistent with previous studies reporting very low levels of IL- $1 \beta$ and no increase in TNF- $\alpha$ plasma levels with treatments below $12.5 \mathrm{mg} / \mathrm{kg}$ bw DON [30,31]. 


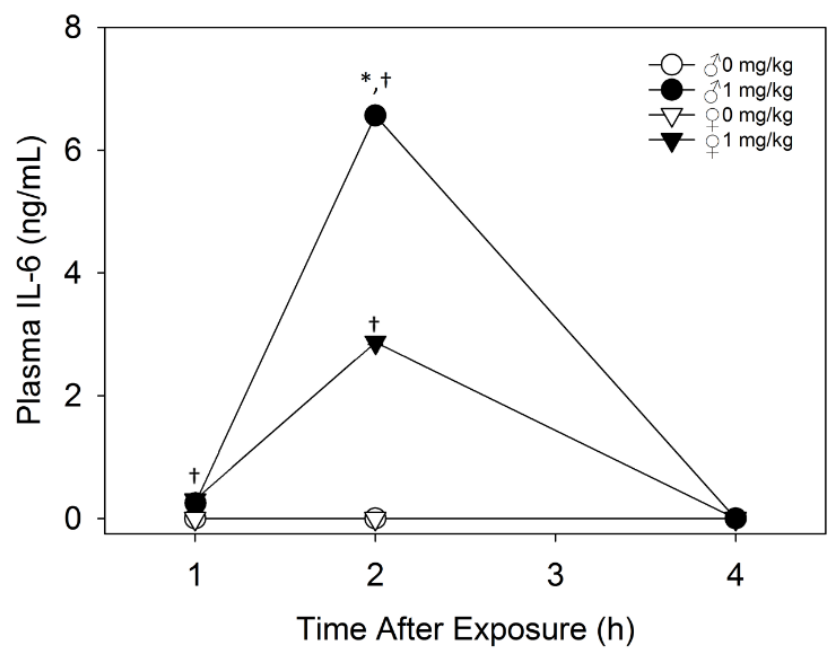

Figure 3. Male mice exhibit higher IL-6 plasma levels at $2 \mathrm{~h}$ post-acute i.p. exposure to $1 \mathrm{mg} / \mathrm{kg}$ bw DON. Data are mean $\pm \operatorname{SEM}(n=4 / \mathrm{rep})$. Asterisk indicates statistical significance from female at time point and dagger indicates significance from group control at time point $(p<0.05)$.

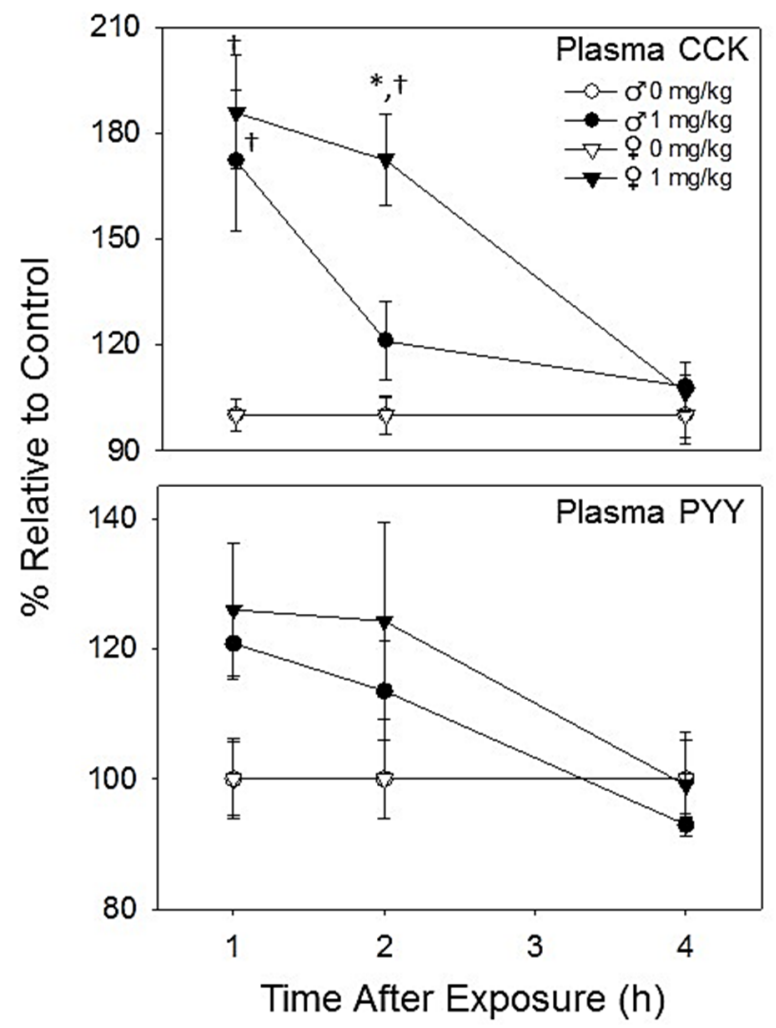

Figure 4. DON induces plasma CCK and PYY elevation. All values are reported as percent of the group control as control females had higher baseline levels of both gut satiety hormones. Data are mean \pm SEM $(n=5-6 / g p)$. Asterisk indicates statistical significance from male at time point and dagger indicates significance from group control at time point $(p<0.05)$. 


\subsubsection{Plasma CCK and PYY Are Increased in Both Female and Male Mice with DON Exposure}

DON treatment increased plasma CCK and PYY concentrations in both male and female mice in comparison to control animals at 1 and $2 \mathrm{~h} \mathrm{PI}$, returning to baseline levels by $4 \mathrm{~h}$ PI (Figure 4). Relative levels of gut satiety hormones in DON-treated female mice tended to be slightly higher than male mice, but only plasma CCK at $2 \mathrm{~h}$ being significantly higher in females. Thus, increased sensitivity of male mice to DON-induced feed refusal could not be explained by differences in either of these two satiety hormones.

\subsection{Study 3}

\subsubsection{Initial Food Intake Is Suppressed in Male Mice But Not Females Exposed to Dietary DON}

When food intake was measured during Study 3, DON-fed male mice exhibited significant differences in the amount of food consumed at 1 and 2 days (Table 2). At these two time points there was significant negative correlation between decreasing food consumption with increasing DON treatments. At 2 days of treatment, males on diets containing 2.5 and $10 \mathrm{ppm}$ DON were eating $15 \%$ and $25 \%$ less respectively than control animals. Translating such decrease to a $2000 \mathrm{kcal}$ diet would correspond in reduction of eating 1700 and 1500 calories, respectively. In comparison, the females did not display a significant suppression in food intake. While female mice showed a trend of decreasing food consumption on day 1 of treatment, the correlation was only approaching statistical significance. After 2 days, mice fed diets containing DON progressively began to shred the food pellets precluding accurate food recovery after this time point.

Table 2. Food consumption is decreased in male mice fed DON containing diets. Values are percent of group control food intake. Data are mean $\pm \operatorname{SEM}(n=6 /$ group$)$.

\begin{tabular}{|c|c|c|}
\hline Group & $\%$ Control Food Intake 0-24 h & \% Control Food Intake 24-48 h \\
\hline$\widehat{o} \mathbf{1} \mathbf{p p m}$ & $99.5 \pm 3.8 *$ & $95.2 \pm 7.2 *$ \\
\hline 2.5 ppm & $85.7 \pm 7.3 *$ & $85.0 \pm 4.9 *$ \\
\hline đ̧ 10 ppm & $56.9 \pm 10.2 *$ & $74.9 \pm 5.6 *$ \\
\hline$\uparrow \mathbf{1} \mathbf{p p m}$ & $100.7 \pm 4.2$ & $95.6 \pm 4.6$ \\
\hline q 2.5 ppm & $99.5 \pm 8.1$ & $101.4 \pm 5.9$ \\
\hline $\mathbf{1 0}_{\mathbf{~ p p m}}$ & $84.0 \pm 7.8$ & $92.3 \pm 8.0$ \\
\hline
\end{tabular}

* indicates statistical significance in correlations between increasing dose and decreasing food intake at time point $(p<0.05)$. Correlation coefficients were: (males 0-24 h; $r=-0.731 ; p=0.00009$ ), (females 0-24 h; $r=-0.384, p=0.06$ ), (males $24-48 \mathrm{~h} ; r=-0.495 ; p=0.01$ ), and (females $24-48 \mathrm{~h} ; r=-0.16 ; p=0.46$ ).

The greater suppression of food intake with dietary DON exposure in male mice compared to females was consistent with the observation of an increased anorectic effect in males versus females in our acute i.p. DON exposure Study 1. These results also correspond to sex differences in food intake with DON exposure that have previously been reported [4,13,15]. Studies in ICR and B6C3F1 mice reported a greater decrease in male food intake with dietary DON exposure when compared to females [4,13]. A meta-analysis performed in swine research also reported that food intake with DON exposure was suppressed in males compared females, with reductions of $20 \%$ and $3 \%$, respectively [15]. 


\subsubsection{Dietary DON Exposure Causes Greater Suppression of Weight Gain in Male Mice}

When the effect of dietary exposure to DON at $0,1,2.5$, and $10 \mathrm{ppm}$ on body weight were compared over 17 days, male mice showed a significant decrease in body weight while female mice did not (Figure 5; Table 3). Males fed 10 ppm DON exhibited significantly suppressed weight gain in comparison to control males and females fed $10 \mathrm{ppm}$ DON diet beginning at day 3 of treatment and maintained this suppression until the end of the experiment. At the termination of the study, males fed with 10 ppm DON diet weighed 17\% less than group controls. After 17 days of exposure to the diet containing $10 \mathrm{ppm}$ DON, female mice exhibited only a $6 \%$ depression of body weight gain compared to group controls. Males on diets containing 1 and $2.5 \mathrm{ppm}$ DON weighed $7 \%$ less than group at the end of the study, while females on these diets weighed only weighed $2 \%$ less than group controls. Adaptation to the toxin and increased metabolic capacity of the toxin are possible explanations for weight loss leveling near the end of the study.

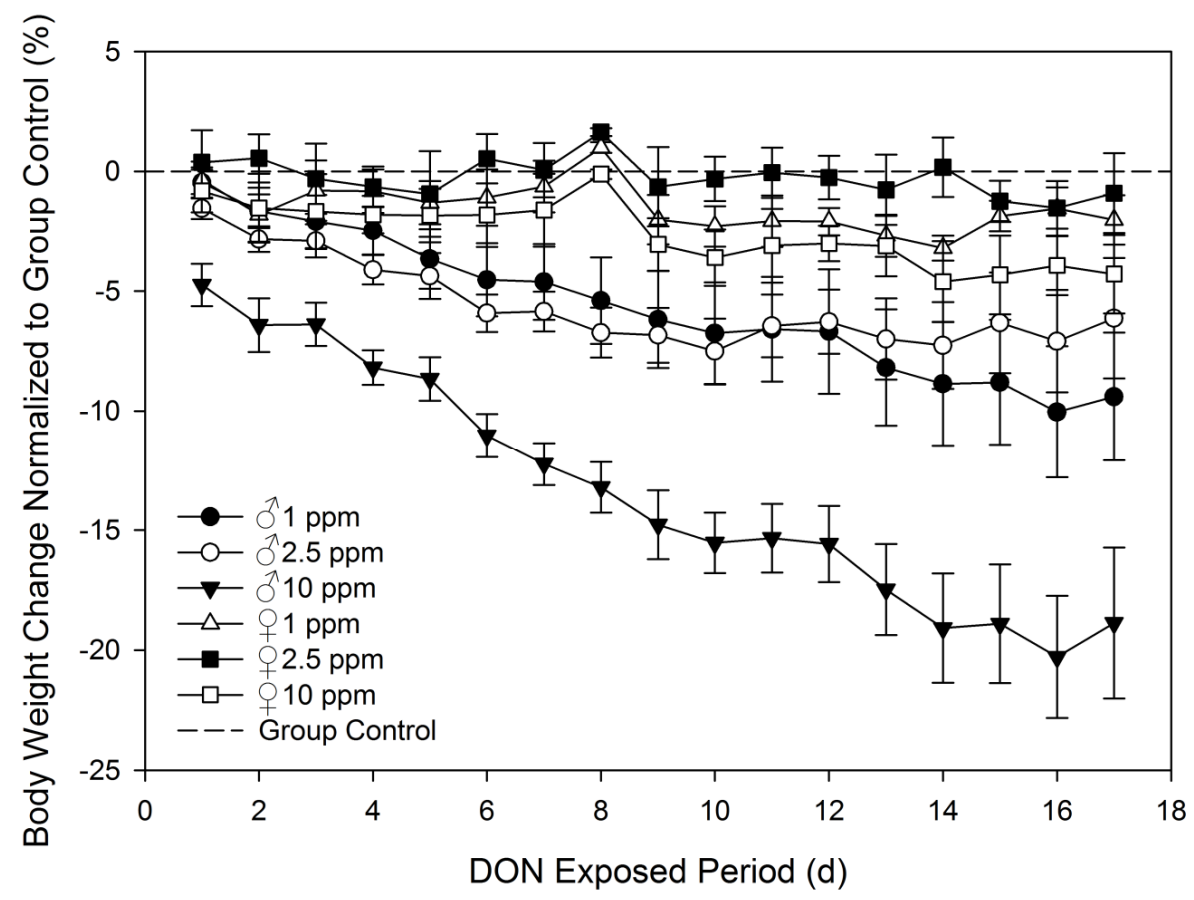

Figure 5. Male mice are more sensitive to DON-induced anorexia than female mice upon dietary DON exposure. Changes in daily body weights were determined from weight at study beginning and normalized to group control weight gain. Data are mean \pm SEM ( $n=6$ /group). Statistical significance is indicated in Table 2.

The results presented here are consistent with previous animal studies examining sex differences with dietary DON exposure [4,11-13,15]. In a study conducted by Cote et al. [12] in swine, researchers found that castrated male swine (barrows) on diets containing $3.1 \mathrm{ppm}$ and $5.8 \mathrm{ppm}$ DON had increased difficulty with consistent weight gain in comparison with female swine. That study also reported that male swine did not resume normal growth rates after being removed from treatment diets, while female swine growth rates recovered. A meta-analysis found an average of weight reduction of $34 \%$ in DON treated barrows, while female weights were only reduced by $2 \%$ [15]. Rotter et al. [13] found that male ICR mice on diets containing 2-8 ppm DON showed a greater suppression of weight gain than females 
over a 14 days period compared to control animals. They also reported that animals appeared to show adaptation to the DON diets in the second week of exposure. A two-year feeding study conducted by Iverson et al. [4] examined sex differences to prolonged DON exposure in B6C3F1 mice. While they did not observe greater weight suppression in male mice compared to female mice, the study did report that males fed diets containing 5 and 10 ppm DON did consume significantly less than control males and no significant differences in food consumption were reported in female mice.

Table 3. Statistical analysis of Figure 5 data. Significant differences are indicated as follows: $\mathrm{a}=$ different from group control body weight percent change and $\mathrm{b}=$ different from female body weight percent change at same dose and day $(p<0.05)$. No statistical significance was observed in females treated with DON in comparison to group control.

\begin{tabular}{|c|c|c|c|c|c|c|c|c|c|}
\hline \multirow[t]{2}{*}{ Treatment } & \multicolumn{9}{|c|}{ Day } \\
\hline & 0 & 1 & 2 & 3 & 4 & 5 & 6 & 7 & 8 \\
\hline đ $1 \mathrm{ppm}$ & - & - & - & - & - & - & - & - & $a, b$ \\
\hline ऽ $2.5 \mathrm{ppm}$ & - & - & - & - & - & - & - & - & $a, b$ \\
\hline$\lesssim 10 \mathrm{ppm}$ & - & - & - & $\mathrm{a}, \mathrm{b}$ & $\mathrm{a}, \mathrm{b}$ & $\mathrm{a}, \mathrm{b}$ & $\mathrm{a}, \mathrm{b}$ & $\mathrm{a}, \mathrm{b}$ & $\mathrm{a}, \mathrm{b}$ \\
\hline & 9 & 10 & 11 & 12 & 13 & 14 & 15 & 16 & 17 \\
\hline$\widehat{\jmath} 1 \mathrm{ppm}$ & $\mathrm{a}$ & $\mathrm{a}, \mathrm{b}$ & - & $a, b$ & $\mathrm{a}$ & - & - & $a, b$ & $\mathrm{a}$ \\
\hline ऽ $2.5 \mathrm{ppm}$ & $a, b$ & $a, b$ & - & $a, b$ & - & - & - & - & - \\
\hline ऽ $10 \mathrm{ppm}$ & $a, b$ & $a, b$ & $\mathrm{a}, \mathrm{b}$ & $a, b$ & $\mathrm{a}, \mathrm{b}$ & $\mathrm{a}, \mathrm{b}$ & $\mathrm{a}, \mathrm{b}$ & $\mathrm{a}, \mathrm{b}$ & $\mathrm{a}, \mathrm{b}$ \\
\hline
\end{tabular}

\subsubsection{Dietary DON Exposure Contributes to Elevated Liver DON Equivalents in Male Mice}

DON equivalents in the organs and plasma after 17 days of dietary DON exposure were compared in male and female mice. Liver DON equivalents in male mice fed 1 and 10 ppm DON diets were statistically significantly higher than females by 2.9 and 1.5 fold, respectively (Figure 6). DON equivalents in the livers of males fed 2.5 ppm DON diets were 1.6 fold higher than the concentrations found females, though this difference was not statistically significant. Liver DON equivalents significantly increased with diets containing higher amounts of DON in both male and female mice. Sex differences in toxin concentrations in all other organs analyzed were not statistically significant, with the exception of kidney DON equivalent concentrations being higher in females than males fed 1 ppm DON diets (Supplementary Data, Figure S2). As animals were allowed ad libitum access to food prior to euthanasia, the amount of toxin consumed and time of consumption are unknown. While unrestricted access to feed prior to euthanasia complicates data interpretation, identifying higher levels of DON equivalents in male mouse livers in both the acute i.p. exposure Study 1 and Study 3 could also suggest that male mice could absorb more toxin and/or require more time to metabolize the toxin. 


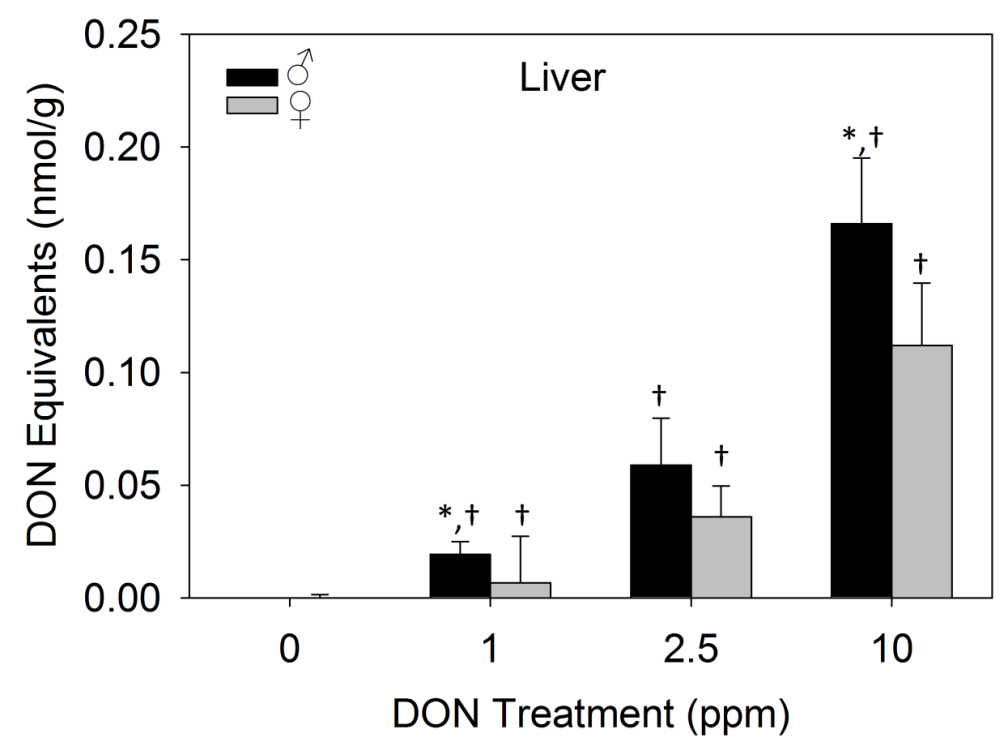

Figure 6. Male mice have higher liver DON equivalents than females after dietary toxin exposure. Data are mean $\pm \operatorname{SEM}(n=6 / \mathrm{gp})$. Asterisk indicates statistical significance from female on same treatment diet and dagger indicates significance from preceding treatment dose within sex $(p<0.05)$.

2.3.4. Proinflammatory Plasma Cytokines Were not Detectable after 17 days of Dietary DON Exposure

Plasma levels of the proinflammatory cytokines IL-6, IL-1 $\beta$, and TNF- $\alpha$ were not detectable in either male or female mice after 17 days of dietary DON exposure at any treatment level. These findings are consistent with a previous study also reporting no detection of plasma levels of IL-6, IL-1 $\beta$, or TNF- $\alpha$ in mice exposed to $2 \mathrm{mg} / \mathrm{mL}$ DON in drinking water for 36 days [32].

\section{Experimental Section}

\subsection{Animals}

The Institutional Animal Care and Use Committee at Michigan State University approved all animal experiments. Adult male and adult female C57BL6 mice (12 weeks) were purchased from Charles River Laboratories (Portage, MI, USA). Mice were housed singly in polycarbonate cages with sifted aspen bedding on a $12 \mathrm{~h}$ light/dark cycle, with constant temperature $\left(21-24{ }^{\circ} \mathrm{C}\right)$ and humidity (40\%-55\%). In all experiments, mice were fed high fat pellet diet (45\% kcal from fat; Research Diets, Inc., New Brunswick, NJ, USA) 1 week prior to DON exposure. High fat diet was used as it was previously determined to provide the most efficient food recovery [10].

\section{2. $D O N$}

DON used for i.p. injections and DON amended diet was obtained from Dr. Tony Durst (University of Ottawa, Canada) and purity was verified to be $98 \%$ by elemental analysis (Galbraith Labs, Knoxville, TN, USA). For all i.p. injections, DON was appropriately dissolved in Dulbecco's phosphate buffered saline (PBS; Sigma-Aldrich, St. Louis, MO, USA) to yield $100 \mu \mathrm{L}$ injection volumes. High fat pellet diets containing $0,1,2.5$ and 10 ppm DON were formulated by Research Diets, Inc. DON concentration 
of diets were confirmed using the Veratox high sensitivity (HS) enzyme-linked immunosorbent assay according to manufacturer protocol (ELISA; Neogen, Lansing, MI, USA).

\subsection{Experimental Design}

\subsubsection{Study 1}

The effects of sex on food intake after acute i.p. DON exposure were assessed, as illustrated in Figure 1. Upon arrival, mice were acclimated to food and handling according to our previously described feed refusal assay [10]. After acclimation, male and female mice ( $n=10 /$ group) were fasted from 10:00 a.m. to 6:00 p.m., and i.p. injected with either 0 (PBS vehicle control), 1 or $5 \mathrm{mg} / \mathrm{kg}$ bw DON (Figure 1). Food consumption was measured hourly 1 to $7 \mathrm{~h}$ post exposure, and at 12, 20, 24 and $36 \mathrm{~h}$ post exposure. Measurements were conducted under red light conditions during dark cycle.

\subsubsection{Study 2}

The effects of sex on tissue DON equivalent concentrations and plasma concentrations of proinflammatory cytokines and satiety hormones were measured after acute i.p exposure to the toxin. Male and female mice ( $n=5-6 /$ group), were similarly fasted as in Study 1 and exposed to $1 \mathrm{mg} / \mathrm{kg}$ bw DON in PBS or PBS vehicle via i.p. injection. Mice were euthanized via $\mathrm{CO}_{2}$ chamber at 1, 2, and $4 \mathrm{~h}$ post exposure without food replacement. Blood was collected via cardiac puncture and the kidney, liver, spleen, heart and brain were collected and immediately snap frozen. Plasma was isolated from blood by centrifugation at $3500 \times \mathrm{g}$ for 10 minutes at $4{ }^{\circ} \mathrm{C}$. Plasma and organs were stored at $-80^{\circ} \mathrm{C}$ until analysis.

\subsubsection{Study 3}

The effects of sex on response to dietary DON exposure were assessed. After acclimation, male and female were randomized into equal weight groups by sex ( $n=6 /$ group) and placed on high fat diets containing 0, 1, 2.5 and 10 ppm DON. Body weights were measured daily at 10:00 am for 17 days. The study was terminated at 17 days as body weight changes appeared to be stabilizing at this point. Food intake measurements were attempted the entire 17 days period. However, after 2 days, mice on diets containing DON progressively began to shred the pellets into fine particles that were unrecoverable from sieved bedding and precluded accurate food recovery. After 17 days of DON exposure, mice were euthanized via $\mathrm{CO}_{2}$ chamber at 8:00 a.m. Food access to treatment diets was ad libitum prior to euthanasia. Blood was collected via cardiac puncture and the kidney, liver, spleen, heart and brain were collected and immediately snap frozen. Plasma was isolated from blood. Plasma and organs were stored at $-80{ }^{\circ} \mathrm{C}$ until analysis.

\subsection{Analytical}

\subsubsection{DON Quantification}

DON quantification in plasma and organs were analyzed using the Veratox high sensitivity (HS) ELISA (Neogen, Lansing, MI, USA) as described previously with slight modifications [26]. Briefly, organs were homogenized 1:1 in PBS (except the heart, which was homogenized 1:2). 
Tissue homogenates were heated at $100{ }^{\circ} \mathrm{C}$ for $5 \mathrm{~min}$ and then centrifuged at $14,000 \mathrm{~g}$ for $10 \mathrm{~min}$ at $4{ }^{\circ} \mathrm{C}$. The resulting supernatant was analyzed for DON using a F3 ELISA plate reader at $650 \mathrm{~nm}$ and Softmax software (Molecular Devices, Menlo Park, CA, USA). DON is reported as DON equivalents as the ELISA was found to be completely cross-reactive with DON3GlcA obtained from Dr. Philipp Fruhmann (Vienna University of Technology, Austria) (Supplementary Data, Figure S1). DONGlcA-3 is the major metabolite formed in rat liver. Cross reactivity with other potential glucuronides was not determined. Since we did not measure ratio of active $v s$. conjugated DON, results are reported as DON equivalents.

\subsubsection{Proinflammatory Cytokine Analyses}

Plasma levels of the proinflammatory cytokines IL-6, IL-1 $\beta$, and TNF- $\alpha$ were determined using Duoset ELISAs from R\&D Systems (Minneapolis, MN, USA). Equal volumes of mouse plasma samples ( $n=5-6 /$ group Study 2; $n=6$ /group Study 3 ) were pooled within groups and ran in technical replicates $(n=4$ rep) because volumes of individual samples were low.

\subsubsection{Satiety Hormone Analyses}

Plasma levels of the gut satiety hormones CCK and PYY $3-36$ in with plasma samples from Study 2 ( $n=6$ /group) were analyzed using ELISA kits for CCK (CCK $26-33$, nonsulfated; human-, rat-, and mouse-specific) and PYY (PYY3-36; mouse-, rat-, porcine-, and canine-specific) (Phoenix Pharmaceuticals, Burlingame, CA, USA). CCK and PYY were not measured in Study 3 as animals had ad libitum access to food prior to study termination.

\subsection{Statistical Analysis}

Statistical analysis was conducted by SigmaPlot version 11.0 (Jandel Scientific; San Rafael, CA, USA, 2009). Statistical comparisons between sexes made at each time point using a Student's t-test, unless normality failed. If normality was not met, a Mann-Whitney Rank Sum test was performed. Statistical comparisons between sex and dose were made at each time point using a one-way analysis of variance (ANOVA), unless normality failed. A Kruskal-Wallis one-way analysis of variance by ranks was performed if normality was not met. Student-Newman-Keuls was used in all post-hoc analysis for parametric and non-parametric animal groups of equal numbers. Dunn's post-hoc analysis was used in non-parametric analysis of animal groups with unequal numbers. Pearson product movement correlations were performed to determine statistical significance between food consumption and treatment levels of DON diets by sex and day in Study 3. Differences were considered significant when $p<0.05$.

\section{Conclusions}

The results presented in this study indicated that male mice were more sensitive than females to anorexia induction after acute and dietary DON exposure. These effects corresponded to decreasing body weight in males fed DON containing diets. As toxin organ clearance was slower in male mice than female mice, future studies should evaluate possible sex differences in DON uptake, metabolism and 
excretion. Relating sex differences to increased vulnerability to DON will be an important consideration in future risk assessment of this and other trichothecenes.

\section{Supplementary Materials}

Supplementary materials can be accessed at: http://www.mdpi.com/2072-6651/7/8/2845/s1.

\section{Acknowledgments}

This study was supported by USDA Wheat and Scab Initiative Award 59-0206-9-058 andPublic Service Grant ES003358 from the National Institute of Health. We would also like to thank Dr. Philipp Fruhmann from Vienna University of Technology for providing us with DON3GlcA standard.

\section{Author Contributions}

Erica Clark contributed to overall design, conduct, and analysis of experiments as well as writing this manuscript. Brenna Flannery contributed to design, conduction of acute exposure studies as well as writing this manuscript. James Pestka contributed to overall design, experimental analysis, interpretation and writing this manuscript.

\section{Conflicts of Interest}

The authors declare that there are no conflicts of interest.

\section{References}

1. Pestka, J.J. Deoxynivalenol: Mechanisms of action, human exposure, and toxicological relevance. Arch. Toxicol. 2010, 84, 663-679.

2. Rodrigues, I.; Naehrer, K. A three-year survey on the worldwide occurence of mycotoxins in feedstuffs and feed. Toxins 2012, 4, 663-675.

3. Trenholm, H.L.; Hamilton, R.M.G.; Friend, D.W.; Thompson, B.K.; Hartin, K.E. Feeding trials with vomitoxin (deoxynivalenol)-contaminated wheat - effects on swine, poultry, and dairy-cattle. J. Am. Vet. Med. Assoc. 1984, 185, 527-531.

4. Iverson, F.; Armstrong, C.; Nera, E.; Truelove, J.; Fernie, S.; Scott, P.; Stapley, R.; Hayward, S.; Gunner, S. Chronic feeding study of deoxynivalenol in B6C3F1 male and female mice. Teratog. Carcinog. Mutagen. 1995, 15, 283-306.

5. Amuzie, C.J.; Pestka, J.J. Suppression of insulin-like growth factor acid-labile subunit expression-a novel mechanism for deoxynivalenol-induced growth retardation. Toxicol. Sci. 2010, $113,412-421$.

6. Voss, K.A. A new perspective on deoxynivalenol and growth suppression. Toxicol. Sci. 2010, 113, 281-283.

7. Wu, W.D.; Bates, M.A.; Bursian, S.J.; Link, J.E.; Flannery, B.M.; Sugita-Konishi, Y.; Watanabe, M.; Zhang, H.B.; Pestka, J.J. Comparison of emetic potencies of the 8-ketotrichothecenes deoxynivalenol, 15-acetyldeoxynivalenol, 3-acetyldeoxynivalenol, fusarenon x, and nivalenol. Toxicol. Sci. 2013, $131,279-291$. 
8. Forsyth, D.M.; Yoshizawa, T.; Morooka, N.; Tuite, J. Emetic and refusal activity of deoxynivalenol to swine. Appl. Environ. Microbiol. 1977, 34, 547-552.

9. Forsell, J.H.; Witt, M.F.; Tai, J.H.; Jensen, R.; Pestka, J.J. Effects of 8-week exposure of the B6C3F1 mouse to dietary deoxynivalenol (vomitoxin) and zearalenone. Food Chem. Toxicol. 1986, 24, 213-219.

10. Flannery, B.M.; Wu, W.D.; Pestka, J.J. Characterization of deoxynivalenol-induced anorexia using mouse bioassay. Food Chem. Toxicol. 2011, 49, 1863-1869.

11. Greene, D.M.; Bondy, G.S.; Azconaolivera, J.I.; Pestka, J.J. Role of gender and strain in vomitoxin-induced dsyregulation of iga production and iga nephropathy in the mouse. J. Toxicol. Environ. Health 1994, 43, 37-50.

12. Cote, L.M.; Beasley, V.R.; Bratich, P.M.; Swanson, S.P.; Shivaprasad, H.L.; Buck, W.B. Sex-related reduced weight gains in growing swine fed diets containing deoxynivalenol. J. Anim. Sci. 1985, 61, 942-950.

13. Rotter, B.A.; Thompson, B.K.; Rotter, R.G. Optimization of the mouse bioassay for deoxynivalenol as an alternative to large animal studies. Bull. Environ. Contam. Toxicol. 1994, 53, 642-647.

14. Greene, D.M.; Azcona-Olivera, J.I.; Pestka, J.J. Vomitoxin (deoxynivalenol)-induced iga nephropathy in the B6C3F1 mouse - dose-response and male predilection. Toxicology 1994, 92, 245-260.

15. Andretta, I.; Kipper, M.; Lehnen, C.R.; Hauschild, L.; Vale, M.M.; Lovatto, P.A. Meta-analytical study of productive and nutritional interactions of mycotoxins in growing pigs. Animal 2012, 6 , 1476-1482.

16. Greene, D.M.; Azcona-Olivera, J.I.; Murtha, J.M.; Pestka, J.J. Effects of dihydrotestosterone and estradiol on experimental IgA nephropathy induced by vomitoxin. Fundam. Appl. Toxicol. 1995, 26, 107-116.

17. Dantzer, R. Cytokine-induced sickness behavior: Mechanisms and implications. In Role of Neural Plasticity in Chemical Intolerance, Sorg, B.A., Bell, I.R., Eds.; 2001; Volume 933, pp. 222-234.

18. Lebrun, B.; Tardivel, C.; Félix, B.; Abysique, A.; Troadec, J.-D.; Gaigé, S.; Dallaporta, M. Dysregulation of energy balance by trichothecene mycotoxins: Mechanisms and prospects. Neurotoxicology 2015, 49, 15-27.

19. Girardet, C.; Bonnet, M.S.; Jdir, R.; Sadoud, M.; Thirion, S.; Tardivel, C.; Roux, J.; Lebrun, B.; Mounien, L.; Trouslard, J.; et al. Central inflammation and sickness-like behavior induced by the food contaminant deoxynivalenol: A PGE2-independent mechanism. Toxicol. Sci. 2011, 124, 179-191.

20. Azcona-Olivera, J.I.; Ouyang, Y.; Murtha, J.; Chu, F.S.; Pestka, J.J. Induction of cytokine messenger-RNAs in mice after oral-exposure to the trichothecene vomitoxin (deoxynivalenol) - relationship to toxin distribution and protein-synthesis inhibition. Toxicol. Appl. Pharmacol. 1995, 133, 109-120.

21. Pestka, J.J.; Amuzie, C.J. Tissue distribution and proinflammatory cytokine gene expression following acute oral exposure to deoxynivalenol: Comparison of weanling and adult mice. Food Chem. Toxicol. 2008, 46, 2826-2831. 
22. Flannery, B.M.; Clark, E.S.; Pestka, J.J. Anorexia induction by the trichothecene deoxynivalenol (vomitoxin) is mediated by the release of the gut satiety hormone peptide yy. Toxicol. Sci. 2012, 130, 289-297.

23. Wu, W.D.; Zhou, H.R.; He, K.Y.; Pan, X.; Sugita-Konishi, Y.; Watanabe, M.; Zhang, H.B.; Pestka, J.J. Role of cholecystokinin in anorexia induction following oral exposure to the 8-ketotrichothecenes deoxynivalenol, 15-acetyldeoxynivalenol, 3-acetyldeoxynivalenol, fusarenon $\mathrm{x}$, and nivalenol. Toxicol. Sci. 2014, 138, 278-289.

24. De Lartigue, G.; Dimaline, R.; Varro, A.; Dockray, G.J. Cocaine- and amphetamine-regulated transcript: Stimulation of expression in rat vagal afferent neurons by cholecystokinin and suppression by ghrelin. J. Neurosci. 2007, 27, 2876-2882.

25. Challis, B.G.; Pinnock, S.B.; Coll, A.P.; Carter, R.N.; Dickson, S.L.; O’Rahilly, S. Acute effects of PYY3-36 on food intake and hypothalamic neuropeptide expression in the mouse. Biochem. Biophys. Res. Commun. 2003, 311, 915-919.

26. Pestka, J.J.; Islam, Z.; Amuzie, C.J. Immunochemical assessment of deoxynivalenol tissue distribution following oral exposure in the mouse. Toxicol. Lett. 2008, 178, 83-87.

27. Prelusky, D.B.; Trenholm, H.L. Tissue distribution of deoxynivalenol in swine dosed intravenously. J. Agric. Food Chem. 1991, 39, 748-751.

28. Buckley, D.B.; Klaassen, C.D. Tissue- and gender-specific mrna expression of UDP-glucuronosyltransferases (UGTs) in mice. Drug Metab. Dispos. 2007, 35, 121-127.

29. Buckley, D.B.; Klaassen, C.D. Mechanism of gender-divergent UDP-glucuronosyltransferase mrna expression in mouse liver and kidney. Drug Metab. Dispos. 2009, 37, 834-840.

30. Amuzie, C.J.; Shinozuka, J.; Pestka, J.J. Induction of suppressors of cytokine signaling by the trichothecene deoxynivalenol in the mouse. Toxicol. Sci. 2009, 111, 277-287.

31. Islam, Z.; Pestka, J.J. Role of IL-1 beta in endotoxin potentiation of deoxynivalenol-induced corticosterone response and leukocyte apoptosis in mice. Toxicol. Sci. 2003, 74, 93-102.

32. Choi, B.-K.; Jeong, S.-H.; Cho, J.-H.; Shin, H.-S.; Son, S.-W.; Yeo, Y.-K.; Kang, H.-G. Effects of oral deoxynivalenol exposure on immune-related parameters in lymphoid organs and serum of mice vaccinated with porcine parvovirus vaccine. Mycotoxin Res. 2013, 29, 185-192.

(C) 2015 by the authors; licensee MDPI, Basel, Switzerland. This article is an open access article distributed under the terms and conditions of the Creative Commons Attribution license (http://creativecommons.org/licenses/by/4.0/). 